\title{
SHIPBOARD PREDICTION WITH A REGIONAL FORECAST MODEL
}

\author{
By Allan R. Robinson
}

$\mathrm{R}$ structures in the ocean and progress in the understanding of related physical processes present new opportunities for realistic ocean scientific studies and efficient marine operations. Over the past two decades, typical synoptic structures of energetic mesoscale meanders and eddies have been determined. Some regions of the world ocean, such as the Gulf Stream system are relatively well observed and studied, whereas others, such as the North Atlantic Current system, still require descriptive research. However, modern methods and strategies can yield rapid results when previously unexplored regions are investigated. New instruments, platforms, sampling schemes, and particularly the coverage afforded by satellite-borne sensors are all contributing to the new knowledge of the physical fields. Dynamical studies, modeling, and simulations provide understanding and feedback. Numerical ocean modeling, which is essential for the representation of realistic fields, is developing very rapidly. Critical support is provided by advances in computer software and hardware, including both supercomputers and workstations.

The new opportunities for ocean scientific studies and efficient marine operations involve the estimation of realistic physical fields. Such field estimations include nowcasts, forecasts, and simulations, which are constructed via the assimilation of data into dynamical models. Dynamical adjustment and dynamical interpolation most efficiently exploit available data, and the melded observational and model-based estimates resulting from assimilation are essentially necessary in oceanography. This is because many mesoscale features occur in large-scale domains of interest, making extensive direct mapping (from observations alone) prohibitively demanding of resources. The realistic physical-field estimates (velocity, pressure, temperature, and salinity) may be directly utilized or may serve as the basis of dynamical studies via the investigation of vorticity

A.R. Robinson, Division of Applied Sciences, Harvard University, Cambridge, MA 02138, USA. balances and energy exchanges. Also, very importantly, these fields provide, for the first time on a very substantial and extensive basis, the possibility of interdisciplinary ocean studies and applications. They represent the four-dimensional (space-time) structures that advect, entrap, and mix dissolved and small-particulate matter. These processes control critical aspects of the dynamics of biogeochemical cycles and ecosystems. They are essential elements of any management model relating to pollution control and resource exploitation and conservation. Such physical nowcast and forecast fields provide, for the first time on a substantially realistic basis, the opportunity for three-dimensional range-dependent acoustic propagations and forecasts. We will focus on the latter application in this review.

A dynamical forecast is a real-time estimate of the future state of the ocean obtained by running a dynamical model forward in time. A hindcast is a forecast made later than real time. A nowcast is an estimate of the present state of the ocean based on melding observations and dynamics. New observations may be melded with a previous forecast or may be used to initialize a short dynamical model run for adjustment and interpolation purposes. Real-time nowcasts and forecasts are important for operations at sea, including those of a scientific nature. Mesoscale phenomena are variable and intermittent in space and time. The location and prediction of events and instrument sites can increase significantly the efficiency of resource use. Predictions, accurate enough to be useful and efficient enough to be feasible, must now be performed on a regional basis. Mesoscale space scales range from tens to hundreds of $\mathrm{ki}$ lometers and time scales from days to months. Data requirements cannot now be met on a global or basinwide scale. Even if this were not the case, the intellectual, technical, and methodological basis for phenomenological interpretation and quality control on such large scales is not yet available. In any case, operational domains of interest are usually limited to domains of a few hundreds or thousands of kilometers in extent.

In many instances, it is desirable to perform the regional forecast at sea aboard an operating 
vessel. The vessel itself can gather the requisite initialization and updating data, in situ subsurface data and/or satellite data. The forecaster may be located aboard the vessel. No communication system is required for a stand-alone operation. The advent of powerful small computers, which are easily carried on and off ships, makes shipboard predictions feasible and convenient today.

The remainder of this article describes a flexible and portable regional forecast system, its shipboard use in real time, and its coupling to an acoustic propagation system. A real-time at-sea exercise in the Northeast Atlantic during the summer of 1987 , which involved the shipboard use of Geodetic Earth Orbiting Satellite (GEOSAT) altimetric data, is presented. This exercise provides the basis for a proof-of-concept feasibility demonstration for the fully coupled environmental/acoustical system, with which we conclude.

\section{The Coupled Environmental/Acoustical System}

To carry out acoustic propagation forecasts in realistic range-dependent environments requires the coupling of a physical-field environmentalforecast system to an acoustic-propagation system. The term system is used to emphasize that the forecasts and their associated sensitivities and error characteristics depend upon a number of factors. For each system these include physical and mathematical modeling assumptions, qualitative and quantitative aspects of the data base, and computational algorithms and parameters. Additionally, the scheme for inputting sound speed distributions to the acoustic system from the environmental system's output of temperature, salinity, and pressure introduces dependencies and sensitivities. This section discusses some of the issues and factors affecting the accuracy and sensitivity of the coupled system and its validation.

The environmental system's dynamical model component (O'Brien, 1986) has explicit physics governing the scales of motion resolved by the horizontal and vertical numerical grids. In other words, the continuous dynamical equations of motion are discretized by an algorithm. But there are also physical processes on scales too small to be resolved by the numerical grids. The subgridscale physics governing the unresolved scales is given a parametric representation (Holloway, 1989). The fundamental (Navier-Stokes) fluid dynamical equations of motion are never solved directly for the scales of motion relevant here. Geometrical and dynamical approximations are employed with the constraints of the earth's rotation and of stable stratification introduced. The primitive equation (PE) approximation, which retains general dynamical balances in the horizontal but which is approximately hydrostatic in the vertical, is appropriate for mesoscale motions. Further simplifications are often desirable to gain effi- ciency or to control aspects of the physics. Most mesoscale dynamical phenomena are almost geostrophic, and the quasigeostrophic (QG) approximation provides an accurate time evolution for the flow field. Exceptions are some interactions with steep topography and aspects of intense meandering of strong jets. The representation of subgridscale physics is difficult and complex. Various nonlinear processes and interactions and turbulent effects may be relevant. Distinction needs to be drawn between horizontal processes (or alternatively, processes along constant-density surfaces) and vertical processes (or alternatively, processes across density surfaces). Practice ranges from the use of simple, constant eddy diffusivities to the use of sophisticated representations of turbulent effects and closure hypotheses. Potential acoustic effects, related to the subgridscale assumptions of the dynamical model influencing the field estimates, are not yet known.

In addition to the dynamical model, the environmental system has a statistical model component and a data assimilation scheme. The statistical model must serve to interpolate data and to put the data onto a regular grid. This is usually done by an objective analysis or optimal interpolation scheme, which minimizes some expected error norm (Bretherton et al., 1976; Clancy et al., 1990). Extrapolation, of special importance for the downward extension of satellite observations, may be accomplished on dynamical modes or empirical orthogonal functions (Preisendorfer, 1988). Additionally, feature models which are average synoptic structures (with a few degrees of freedom) are employed to minimize data requirements and to extend satellite observations before assimilation into the dynamical model. Data assimilation has recently entered oceanography and considerable research has been started to determine the advantages and sensitivities of various schemes in various situations of oceanographic interest (Anderson and Willebrand, 1989; Haidvogel and Robinson, 1989). Schemes range from simple optimal interpolation to the more complex variationally based inverse and adjoint methods. Computational efficiency considerations are paramount. The data bases required include the bottom topography, climatology, surface fluxes, and synoptic data for initialization and updating. An observational network with a mix of remotely sensed and in situ data types is usually the most efficient.

The dynamical model of the acoustical system also is usually an approximation to the fundamental Helmhotz equation. Assumptions depend on the frequency range and the specific physics and geometry considered. Ray theory, the parabolic-equation approximation for the far field, and normal modes are frequently used (Potter and Warn-Varnas, 1991). In addition to the four-dimensional sound speed distribution, environ-
. . forecasts and

their associated

sensitivities and error

characteristics depend

upon a number of

factors. 
. . a versatile open-

ocean forecasting

system . . . has been

exercised and

validated at 11 sites in

the world ocean. mental and boundary conditions associated with the sea surface and bottom (and the underlying sediments) can produce a number of phenomena, which may or may not be important and may or may not be explicitly included in the propagation calculation. In considering the coupling, it is important that the environmental model evolve satisfactorily those structural features which affect the acoustics, even though they may not be important for the dynamical evolution of the main mesoscale fronts and eddies. Also, the acoustic model requires a vertical and horizontal grid of sound-speed input of much higher resolution than is necessary or efficient for the running of the ocean dynamical model. Thus interpolation is required and research in relevant methodology is currently underway.

\section{The Forecast System}

Our physical oceanography group has developed a versatile open-ocean forecasting system. The system is flexible and portable both scientifically and logistically. Since 1980 , it has been exercised and validated at 11 sites in the world ocean as shown on the map of Figure 1. All but the original POLYMODE* site involved real-time forecasts, and at six sites the real-time forecasts were carried out aboard ships. Synoptic data input has included temperature and salinity profiles, current measurements, and both sea-surface temperature and height sensed from satellites.

The original dynamical model component is an open-ocean quasigeostrophic model that was developed to study the dynamical processes inherent in local intensive data sets. The approach involved real data initializations of a block of open ocean and thus encompassed the forecast problem. A review of this work, including the system's multivariate objective analysis scheme and the associated energy and vorticity analysis scheme, is presented by Robinson and Walstad (1987). The present forecast system is based on a dynamical model hierarchy. There is a set of models with dynamics and geometry appropriate to various oceanic regions. The QG model has been generalized to domains that may be partially open and partially closed by arbitrary coastlines and may contain islands (Özsoy et al., 1991). A three-dimensional surface-boundary-layer (SBL) model has been coupled to the full water-column eddyresolving model (Walstad, 1987) to represent the details of upper-ocean physics. A primitive-equation open-ocean model for the water column has been developed (Spall and Robinson, 1989) starting from the basic Geophysical Fluid Dynamics Laboratory primitive equation model (Bryan and Cox, 1967). The horizontal subgridscale physics

* A program name derived from the Russian word polygon that means moored array and the US-UK acronym for MidOcean Dynamics Experiment. in both the PE and QG models is a smoothing operation via an arbitrary order Shapiro filter (Robinson and Walstad, 1987). This prevents the build-up of energy in small scales of the numerical grid, which would otherwise occur due to the enstrophy cascade of geostrophic turbulence. Present data assimilation schemes include optimal interpolation and for the QG model, an efficient adjoint (Moore, 1991).

The first real-time forecast was carried out in 1983 in the context of the Ocean Prediction Through Observation, Modeling and Analysis (OPTOMA) program at the Naval Postgraduate School, by radio communication with the $R / V$ Acania, which was gathering data in the California Current (Robinson et al., 1986). In 1984, the dynamical model was first taken to sea on a microcomputer, and used in the Nares Plain region of the Northwest Atlantic. Dr. L.J. Walstad and Mr. W.G. Leslie took it to forecast for real-time experimental guidance (Robinson, 1986). These and related studies led to the development of the modular concept of dynamical model initialization with in situ data being acquired in real time. A full mapping grid is not necessary. Dynamical interpolation can be successfully carried out into the interior from data taken on the boundaries of a square module. Each module is somewhat less on a side than the eddy-decorrelation length scale. A large region can be efficiently built up from a number of adjoining modules with reinitializations and updates. Once the full domain of interest has been initialized, only the boundaries need updating to maintain most open-ocean regional nowcasts and forecasts for a considerable length of time. When satellite or aircraft data are also available, other initialization and updating strategies are more efficient, and even less in situ data is required. Although direct current measurements are desirable, they are not always essential because hydrography or altimetry plus model dynamics can together provide the unmeasured barotropic mode (Robinson et al., 1986; DeMey and Menard, 1989).

The Gulf Stream meander and ring region has been the location of considerable research on dynamics and prediction during the past several years. Starting in 1986, for a period of over 2 years, the group at Harvard carried out with Navy support I-week-duration day-by-day forecasts (Gulfcasts) of the Gulf Stream frontal and ring locations and interactions (Robinson et al., 1989). Initialization and updating nowcasts melded dynamical model forecasts with new observations of frontal locations obtained from satellite infrared sea-surface temperature and GEOSAT altimetric seasurface heights. Also, there was nominally, once a week, a dedicated P3 AXBT flight, i.e., a Navy patrol aircraft dropped $\sim 35$ temperature versus depth probes in specially identified locations. Approximately $75 \%$ of the ring-birth and reabsorp- 


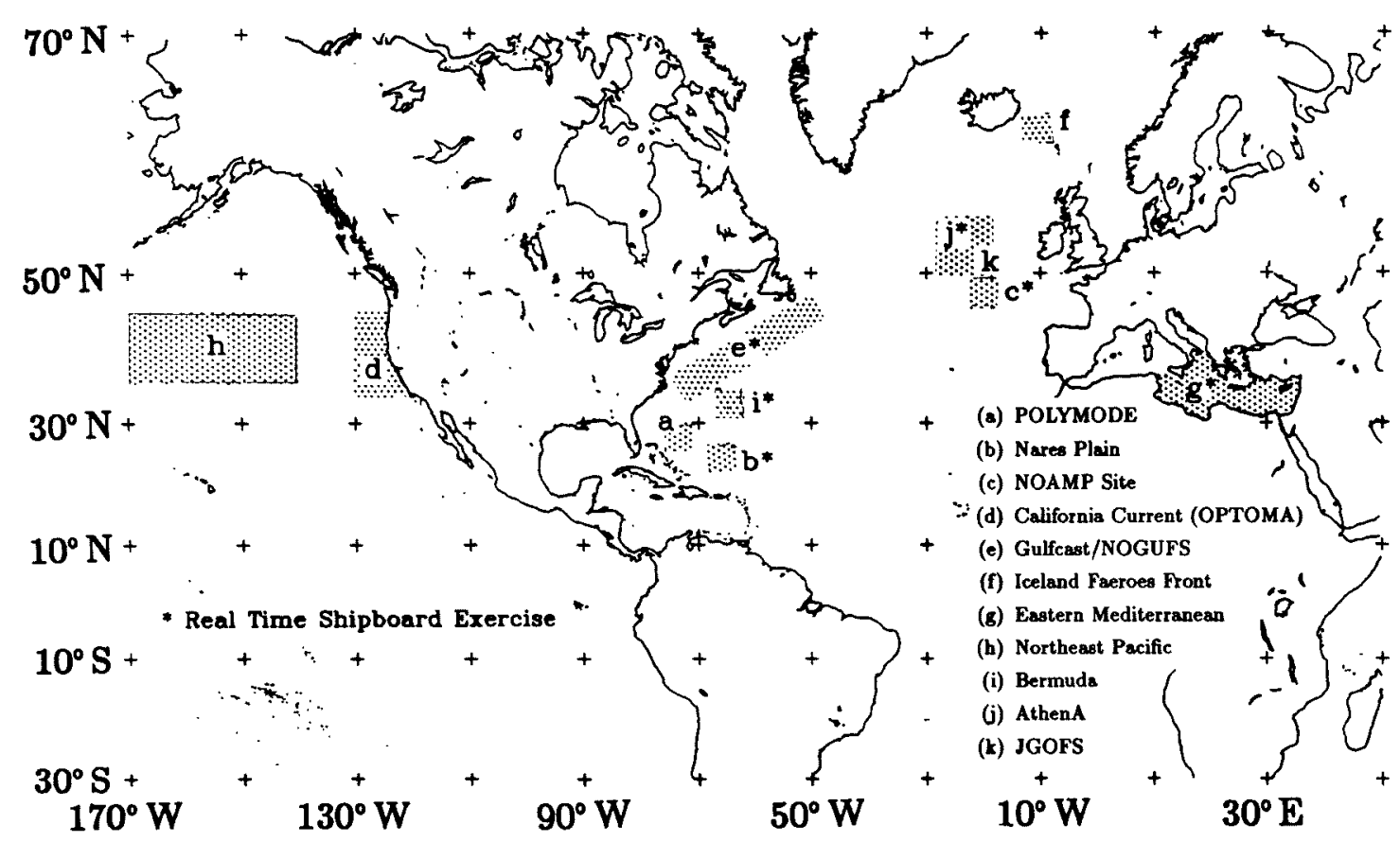

Fig. 1: Locations of operation of the Harvard forecast system. POLYMODE: POLYgon-Mid-Ocean Dynamics Experiment. NOAMP: NOrth Atlantic Monitoring Program. OPTOMA: Ocean Prediction Through Observation, Modeling and Analysis. NOGUFS: Naval Operational GUlf stream Forecast System. AthenA: Acquisition de données de Topographie de surface et d'Hydrologie dans l'Est Nord Atlantique. JGOFS: Joint Global Ocean Flux Study.

tion events were forecast correctly, and a posteriori verification of the Stream frontal location offsets with GEOSAT data (Glenn et al., 1991) yielded a standard deviation of $\sim 30 \mathrm{~km}$, the noise level of the system. In December 1989, the system was transferred for operations to the Naval Oceanographic Office [with the name Naval Operational GUlf stream Forecast System (NOGUFS) ] and was cited by Rear Admiral J. Koehr as "the Navy's first dynamic oceanographic forecast model." The opportunity provided for realistic acoustic propagation studies by the Gulfcast fields resulted in their utilization by acousticians at a number of institutions including the Naval Oceanographic and Atmospheric Research Laboratory, the Naval Underwater Systems Center (NUSC, New London and Newport), the Woods Hole Oceanographic Institution, and the Naval Postgraduate School. In particular, an ongoing collaboration has developed between the Harvard group and Dr. D. Lee of NUSC (New London) and Professor W. Siegmann of Rensselaer Polytechnic Institute in coupling their parabolic equation propagation model, implemented numerically as the Implicit Finite Difference (IFD) model (Lee et al., 1981), to Harvard dynamical model outputs. Sensitivity studies (Siegmann et al., 1990) and fully threedimensional results (Lee et al., 1989) have been obtained.

A two-dimensional version of the IFD model now resides at Harvard. It has been coupled to the output of the full dynamical model hierarchy, $\mathrm{PE}, \mathrm{QG}$, and SBL and completes the present version of the Harvard environmental/acoustical forecast system. Two dimensions are accurate for most propagation situations, with exceptions including regions of strong azimuthal topographic variation (Siegmann et al., 1990). A realistic sediment layer is included and important. Carman (1991) has used this system to study combined oceanographic and topographic effects on acoustic propagation (in regions $\mathrm{e}, \mathrm{f}$, and $\mathrm{j}$ of Fig. 1).

\section{A Real-Time Exercise and Proof-of-Concept for the System}

Site $\mathbf{j}$ (Fig. 1), in the Northeast Atlantic, was the location of a real-time experiment during July and August 1987, carried out on board the French Naval vessel B.O.D'Entrecasteaux. The objectives of the experiment, called Acquisition de données de Topographie de surface et d'Hydrologie dans l'Est Nord Atlantique (AthenA), were related to the methodology of real-time shipboard prediction using a dynamical forecast system and a combination of in situ and remotely sensed data. Harvard scientists participated with the quasigeostrophic model run on one of the group's SUN-3 workstations. The ship received GEOSAT altimetric data in near real time, transferred via Harvard from the Johns Hopkins University Applied Physics Laboratory, which was used for GEOSAT and model validation. The ship also received sat-
. . . "the Navy's first

dynamic

oceanographic forecast

model" . . . provided

for realistic acoustic

propagation

studies ... 


\section{... forecast height}

field compared with an

independent estimate

from a GEOSAT pass

. . The agreement is

again excellent . . . ellite infrared imagery. The experimental domain was an open-square block of water $210 \mathrm{~km}$ on a side with a fairly flat bottom, which contained energetic mesoscale eddies associated with the North Atlantic Current and Drift. Two synoptic realizations of the hydrographic fields were obtained with Conductivity-Temperature-Depth sensors (CTDs) and expendable bathythermographs (XBTs) just over 2 weeks apart. Drifting buoys and current meters provided absolute flow measurements. More details can be found in Carman's (1991) thesis and a French report (LeSquere, 1989).

Shipboard activities included real-time objective analyses and dynamical forecasts with six levels in the QG model. Figure 2A shows the seasurface height field (in meters) associated with the shipboard nowcast of the first synoptic realization centered on 29 July. Hydrography plus drifter data have been assimilated into the dynamical model. The north-south oriented front is the border between two eddies of opposite sign. The $\sim 0.25-\mathrm{m}$ drop across the front corresponds to a southward flowing geostrophic current of $0.60 \mathrm{~m} / \mathrm{s}$ maximum. The diagonal line on Figure $2 \mathrm{~A}$ is the ground track of the GEOSAT satellite. Figure 2C

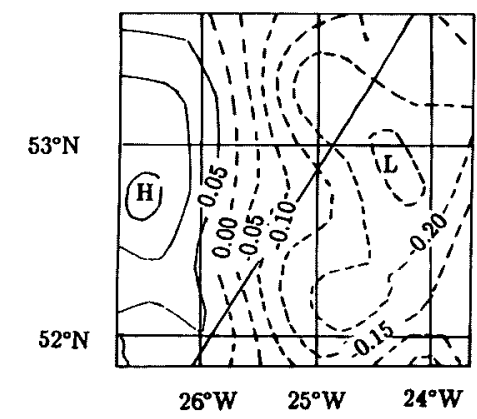

(a)

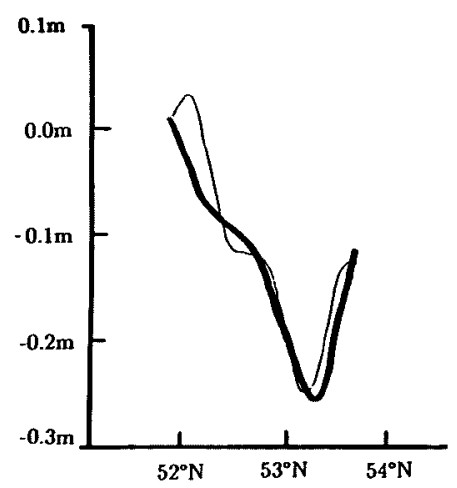

(c)

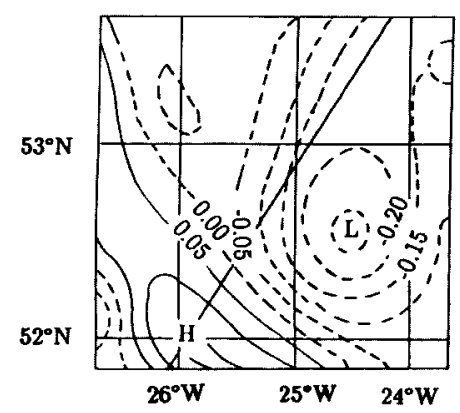

(b)

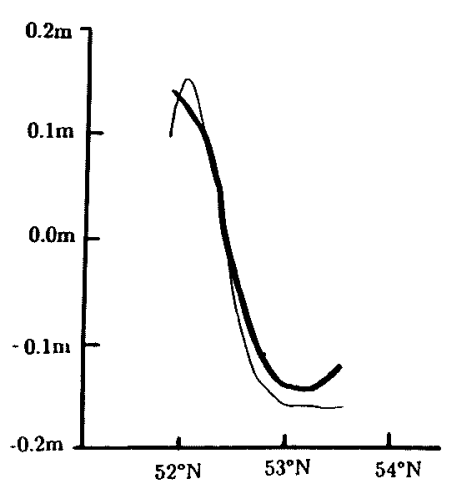

(d)

Fig. 2: (A) Sea-surface height (meters) nowcast for 29 July 1987. (B) Forecast for 15 August 1987. GEOSAT track is the diagonal line. (C) Alongtrack comparisons of a nowcast (fine) and GEOSAT (bold) height estimates. (D) Alongtrack comparisons of $\boldsymbol{B}$ forecast (fine) and GEOSAT (bold) height estimates. Note the axis shift of $0.1 \mathrm{~m}$ between $\mathrm{C}$ and $\mathrm{D}$.

compares two independent along-track estimates of the sea-surface height for 29 July. The first is obtained from the nowcast (fine line), and the second from the altimetric data derived by a method based on subtracting the individual track from the time mean of all the altimetric data along that track. The agreement in shape and amplitude is excellent; the individual track absolute mean altimetric height is not known over such a short segment (Porter et al., 1989). The validation of the GEOSAT altimetric topography is within the noise level of the system. During the next 2 weeks the eddy system evolved with the western cyclone moving to the southeast and the front shifting orientation as shown on Figure 2B. The height field (Fig. 2B) has been forecast for 17.5 days from the nowcast (Fig. 2A) initialization. Only the boundary condition has been updated with new information from hydrography and floats. Figure 2D shows the along-track forecast height field compared with an independent estimate from a GEOSAT pass on 15 August. The agreement is again excellent, which can now be regarded as a GEOSAT validation of the forecast system. Unfortunately GEOSAT data return during the experiment was very poor in the region. However, Dombrowsky and De Mey (1992) have made detailed comparisons of Harvard QG model forecasts with updated boundary conditions and the Athen $A$ in situ data and finds very good agreement throughout the experiment.

For this type of mid-ocean eddy field the QG model is adequately accurate. The types of small differences that occur between PE and QG dynamics have been studied for a Northwestern Atlantic site by Spall (1989). However for domains only a few hundred kilometers on a side, the openocean PE of the Harvard model set is almost as efficient computationally as the QG, and its shipboard use is optional.

Because the AthenA experiment involved realtime forecasting at sea with a mix of remotely sensed and in situ data, it was selected for a proofof-concept feasibility demonstration of the full coupled environmental/acoustical forecast system. The hindcast was carried out using the coupled quasigeostrophic/surface-boundary-layer dynamical models and the two-dimensional IFD parabolic model. The QG used nine levels, and the SBL used eight additional levels. The QG water-column model was run for 10 days, which is a reasonable length of time for main thermocline evolution without the need for boundary updating. The SBL forecast for the upper ocean, which requires an atmospheric forecast for surface flux input, was limited to 3 days, and sound was propagated at 50 and $100 \mathrm{~Hz}$ for various source depths on the third day. The arrows on the 6-m-depth temperature field of Figure $3 \mathrm{~A}$ and the $125-\mathrm{m}$ depth QG streamfunction field of Figure 3B indicate the location of the vertical sections for the propagation calculations. The date is August 3, 
and the thermocline streamfunction field depicted in Figure 3B is proportional to geostrophic pressure or dynamic height (shown for July 31 in Fig. 2A). A difference of 20 nondimensional stream function units produces the geostrophic jet of $\sim 60$ $\mathrm{m} / \mathrm{s}$. Smaller-scale features are evident in the nearsurface temperature. The sound speed section (Fig. $3 C$ ) indicates a sound channel axis, which rises to the east from $\sim 900$ to 500 -m depth. Near-surface detailed structure is evident and the QG/SBL model treatment has increased near-surface sound speeds by $\sim 5 \mathrm{~m} / \mathrm{s}$ over a simple QG model forecast. Propagation in the sediment below the sea bottom included effects of a vertical sound speed gradient and an attenuation factor. An exemplary propagation-loss section is shown in Figure 3D for a $50-\mathrm{Hz}$ source at $500-\mathrm{m}$ depth. Detailed interpretation of the oceanographic effects on propagation are interesting but not the subject of this article. Carman (1991) finds eddy frontal effects of $45 \mathrm{~dB}$. There is a near-surface duct that affects frequencies too low to be trapped. Changes as large as $25 \mathrm{~dB}$ occur relative to comparison calculations made without the SBL model coupled to resolve the near-surface structures. The bottom is nearly flat at just under $4500-\mathrm{m}$ depth, except for a hill in the west. Note the propagation within the sediments, which influences the water-column propagation.

The efficiency of this portable and realistic forecast system can be measured by the clocktime required to produce the acoustic forecasts after the acquisition of nowcast data. The objective analysis took approximately one-half hour of SUN-3 workstation time, the 10-day QG forecast took 1 hour, and the 3-day SBL forecast one-half hour. The IFD propagations were run on an Iris, and 1 hour of clocktime yielded from 2 to 6 source cases dependent on frequency. Thus the system is very efficient with only 3-4 hours being required. Moreover the workstations used are no longer state-of-the-art. Currently available workstations could reduce the time required by a factor of 10 and allow improvements such as increased resolution and more sophisticated assimilation schemes.

\section{Summary and Conclusions}

The ability for the first time in ocean science history to forecast realistic physical fields makes realistic three- and four-dimensional range-dependent acoustical propagation now possible on a substantial basis. The concept of a coupled environmental/acoustical system has been introduced and factors affecting its accuracy discussed. A portable, flexible system that is feasible, accurate, and efficient for shipboard use has been described and its use illustrated in both the forecast and hindcast mode. Realistic real-time shipboard forecasts of practical interest can be achieved in only a few hours of clocktime.

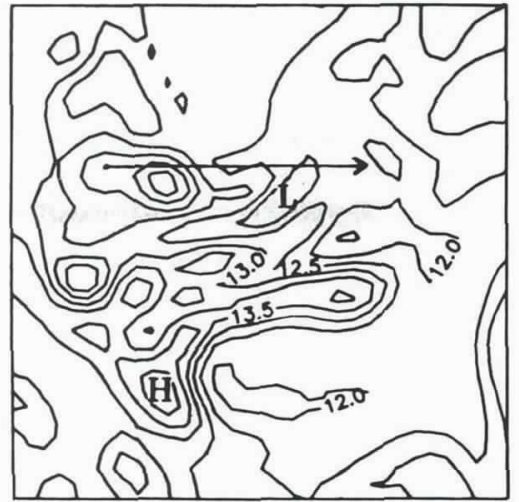

11.4214 .870 .50

(a)

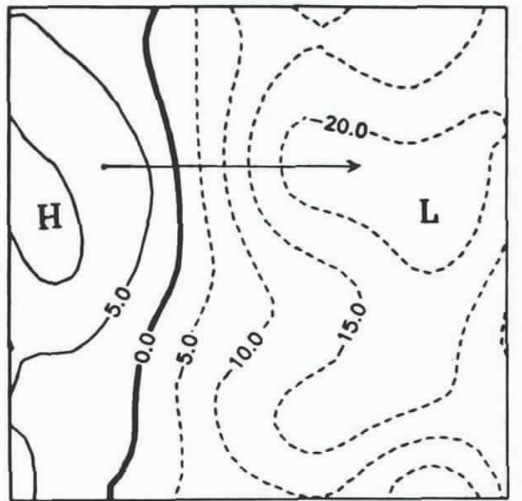

$-23.1711 .985 .00$

(b)

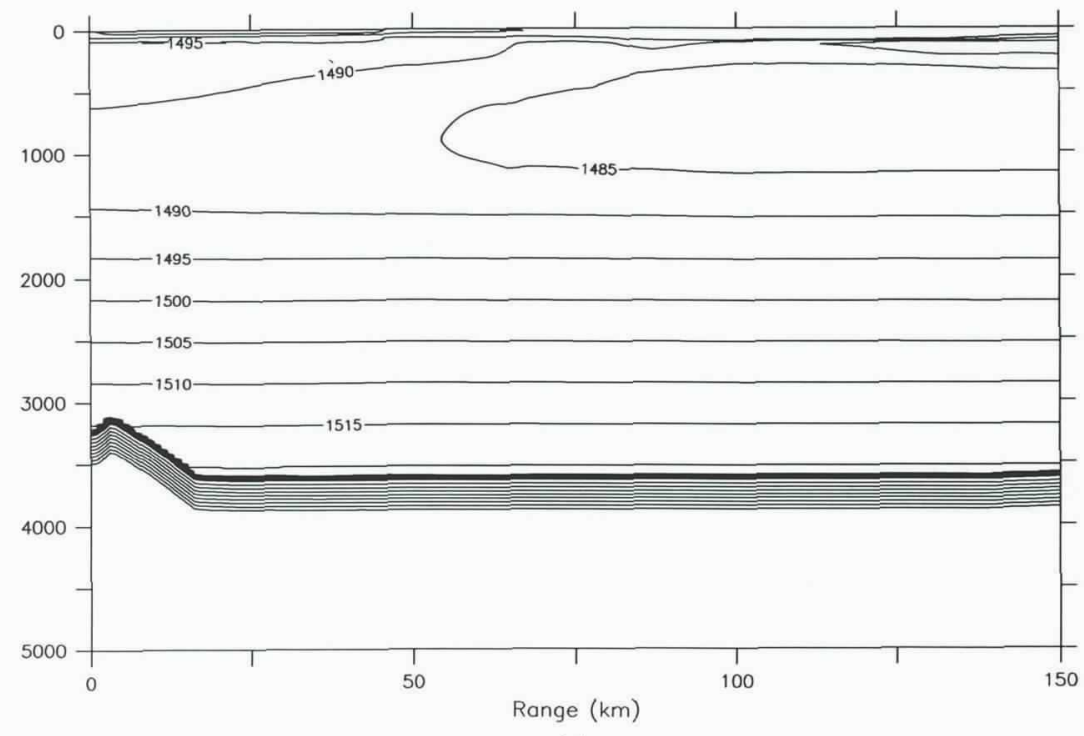

(c)

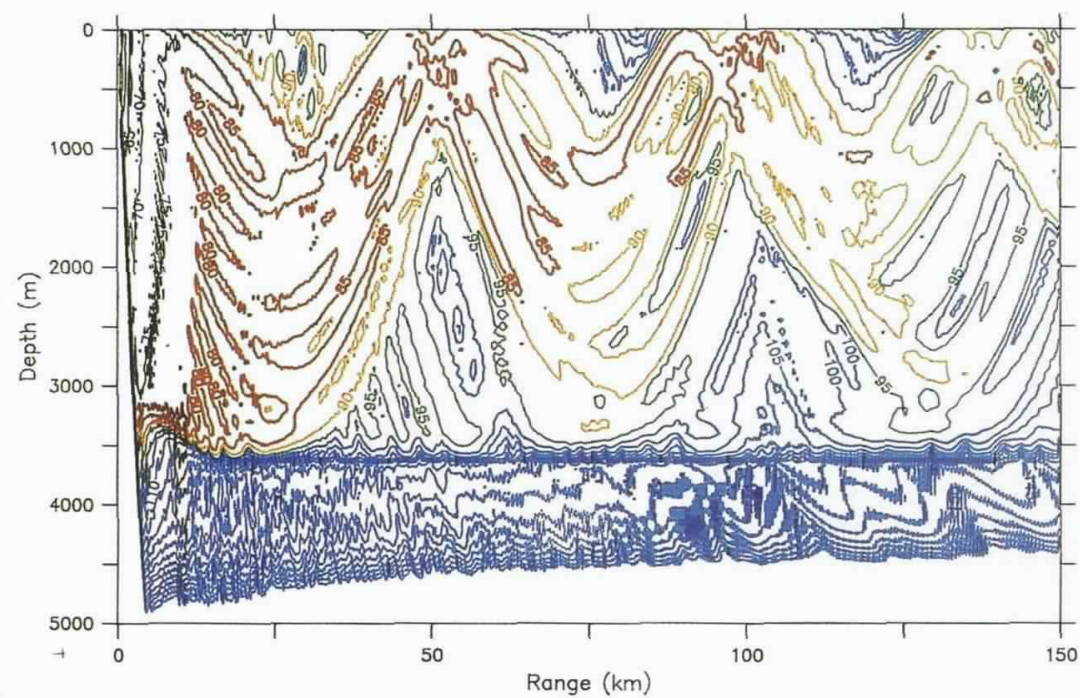

(d)

Fig. 3: Three-day forecasts of (A) temperature at $6 \mathrm{~m}\left(0.5^{\circ} \mathrm{C}\right.$ contours), (B) non-dimensional quasigeostrophic $(Q G)$ streamfunction at $125 \mathrm{~m}$ (arbitrary units; see text), $(\boldsymbol{C})$ sound speed $(\mathrm{m} / \mathrm{s})$ and $(\boldsymbol{D})$ propagation loss (decibels) for a 50-Hz source at $500 \mathrm{~m}$. (Black 50-75 dB; red 80-85 dB; yellow 90-94 dB; green 95-99 dB; blue 100-170 dB). Minima, maxima, and contour intervals are labeled below $\boldsymbol{A}$ and $\boldsymbol{B}$. 


\section{Acknowledgements}

The proof-of-concept hindcast was carried out at Harvard with the help of J.C. Carman, C.J. Lozano, A. Gangopadhyay, and D.J. McGillicuddy. L.J. Walstad, D.W. Denbo, and J.C. Carman performed the real-time nowcasts and forecasts during AthenA, and J. Calman, E.B. Dobson, and D. Porter provided the GEOSAT data. I thank A. Gangopadhyay, M.G. Cormier, W.G. Leslie, and C.J. Lozano for help in the preparation of the manuscript. ONR Support under grants N00014-90-J-1593 and N00014-90-J1612 is gratefully acknowledged. The University Research Initiative contract N00014-86-K-0751 supported (in part) the real time exercises during AthenA.

\section{References}

Anderson, D.L.T, and J. Willebrand, eds., 1989: Oceanic Circulation Models: Combining Data and Dynamics. Kluwer Academic Publishers, Dordrecht, Netherlands, 605 pp.

Bretherton, F.P., R.E. Davis and C.B. Fandry, 1976: A technique for objective analysis and design of oceanographic experiments applied to MODE-73. Deep-Sea Res., 23, $559-582$.

Bryan, K. and M.D. Cox, 1967: A numerical investigation of the oceanic general circulation. Tellus, 19, 54-80.

Carman, J.C., 1991: Oceanographic and topographic interactions in underwater acoustic propagation, with regional applications. Ph.D. thesis, Harvard University, $295 \mathrm{pp}$.

Clancy, R.M., P.A. Phoebus and K.D. Pollak, 1990: An operational global-scale ocean thermal analysis system. $J$. Phys. Oceanogr., 7, 233-254.

DeMey, P. and Y. Menard, 1989: Synoptic analysis and dynamical adjustment of GEO-3 and SEASAT altimeter eddy fields in the Northwest Atlantic. J. Geophys. Res. 94. $6221-6231$.

Dombrowsky, E. and P. DeMey, 1992: Continuous assimilation in an open domain of the Northeast Atlantic, part 1 : methodology and application to AthenA-88. J. Geophys. Res., 97.

Glenn, S.M., D.L. Porter and A.R. Robinson, 1991: A synthetic geoid validation of Geosat Mesoscale Dynamic Topography in the Gulf Stream region. J. Geophys. Res. Occans, 96, 7145-7166.

Haidvogel, D.B. and A.R. Robinson, eds., 1989: Data Assimilation. Dynamics of Atmospheres and Oceans. Special Issue, 13. Elsevier Science Publishers, Amsterdam, Netherlands, $518 \mathrm{pp}$.

Holloway, G., 1989: Subgrid scale representation. In: Oceanic Circulation Models: Combining Data and Dynamics.
D.L.T. Anderson and J. Willebrand, eds., Kluwer Academic Publishers, Dordrecht, Netherlands, 513-593.

Lee, D., G. Botseas and J.S. Papadakis, 1981: Finite-difference solution to the parabolic wave equation. J. Acoust. Soc. Amer., 70, 795-800.

Lee, D., G. Botseas, W.L. Siegmann and A.R. Robinson, 1989: Numerical computations of acoustic propagation through three-dimensional ocean eddies. In: Numerical Mathematics. W.F. Ames, ed., J.C. Baltzer AG, Scientific Publishing, Basel, Switzerland, 317-321.

LeSquere, B., 1989: Rapport sur le traitement des données de la campagne AthenA 88, CNRM/HDC Toulouse.

Moore, A.M., 1991: Data assimilation in a quasigeostrophic open-ocean model of the Gulf Stream region using the adjoint method. J. Phys. Oceanogr., 21, 398-427.

O'Brien, J.J., ed., 1986: Advanced Physical Oceanographic Numerical Modeling. D. Reidel Publishing Company, Dordrecht, Netherlands, $608 \mathrm{pp}$.

Özsöy, E.. C.J. Lozano and A.R. Robinson, 1991: A consistent baroclinic quasigeostrophic ocean model in multiply connected ocean domains. Math. Comp. Sim., 34, $51-$ 79.

Porter, D.L., A.R. Robinson. S.M. Glenn and E.B. Dobson, 1989: The synthetic geoid and estimation of mesoscale absolute topography from altimeter data. Johns Hopkins APL Tech. Digest, 10, 369-379.

Potter. J. and A. Warn-Varnas, 1991: Ocean V'ariability and Acoustic Propagation. Kluwer Academic Publishers, 608 pp.

Preisendorfer, R.W., 1988: Principal Component Analysis in Meteorology and Oceanography. Elsevier Science Publishers, Amsterdam, Netherlands, $425 \mathrm{pp}$.

Robinson, A.R., J.A. Carton, N. Pinardi and C.N.K. Mooers, 1986: Dynamical forecasting and dynamical interpolation: an experiment in the California current. J. Phys. Oceanogr., 16, 1561-1579.

Robinson, A.R., S.M. Glenn, M.A. Spall, L.J. Walstad, G.M. Gardner and W.G. Leslie, 1989: Forecasting Gulf Stream meanders and rings. EOS, 70, 1464-1473.

Robinson, A.R. and L.J. Walstad, 1987: The Harvard open ocean model: calibration and application to dynamical process forecasting and data assimilation studies. Appl. Numer. Math., 3, 89-131.

Siegmann, W.L., M.J. Jacobson, D. Lee, G. Botseas, A.R. Robinson and S.M. Glenn, 1990: Interfacing mesoscale ocean prediction and parabolic acoustic propagation models. In: Computational Acoustics II. D. Lee, A. Cakmak and R. Vichnevetsky, eds., Elsevier Science Publishers, Amsterdam, Netherlands, 155-168.

Spall, M.A., 1989: Regional primitive equation modeling and analysis of the POLYMODE data set. Dyn. Atmos. Oceans, 14, 125-174.

Spall, M.A. and A.R. Robinson, 1989: A new open ocean, hybrid coordinate primitive equation model. Math. Comp. Sim. 31, 241-269.

Walstad, L.J., 1987: Modeling and forecasting deep ocean and near surface mesoscale eddies. Ph.D. thesis, Harvard University, $266 \mathrm{pp}$. 